\title{
Development of a 3D Dynamic Programming Method for Weather Routing
}

\author{
S. Wei \& P. Zhou \\ Department of Naval Architecture and Marine Engineering, University of Strathclyde, \\ Glasgow, UK
}

\begin{abstract}
This paper presents a novel forward dynamic programming method for weather routing to minimize ship fuel consumption during a voyage. Compared with the traditional two dimensional dynamic programming (2DDP) methods which only optimize the ship's heading, while the engine power or propeller rotation speed are set as a constant throughout the voyage, this new method considers both the ship power setting and heading control. A float state technique is used to reduce the iteration on the process of optimization for computing time saving. This new method could lead to a real global-optimal routing in a comparison with a tradition weather routing method which results in a sub-optimal routing.
\end{abstract}

\section{INTRODUCTION}

Ship weather routing is defined as an optimum track of ship route with an optimum engine speed and power for an ocean voyage based on en-route weather forecasts and ship's characteristics. Within specified limits of weather and sea conditions, the term optimum means a maximum of safety and crew comfort, a minimum of fuel consumption and time underway, or any desired combination of these factors. It can be clearly seen that, the accuracy of determining the optimum route depends on three aspects.

- The accuracy of the prediction of the ship's hydrodynamic behavior under different weather conditions.

- The accuracy of the weather forecast.

- The capability and practicability of the optimization algorithm.

The focus of this study is on research of the optimization algorithm. Many optimization algorithms have been developed for solving ship routing problems in which ship fuel consumption and/or passage time are minimized. Most popular methods include calculus of variations (Bijlsma S.J 1975), modified isochrone method (Hagiwara H 1989, Hagiwara H \& Spaans JA 1987), two dimensional dynamic programming (2DDP) method (De Wit C 1990, Calvert
$\mathrm{S}$ et al. 1991,) and isopone method (Klompstra MB et al. 1992, Spaans JA 1995) .

The method of calculus of variation treats the ship routing as a continuous optimization problem. Inaccuracy in the solution may arise in the functions where second order differentials are required. The errors could be expanded to an unacceptable level at the end of the calculation.

The modified isochrone method is a recursive algorithm. The route with the minimum of passage time is obtained by repeatedly computing isochrones (or time fronts) which are defined as the outer boundaries of the attainable region from the departure point after a certain time. This method offers a route with minimum fuel consumption by keeping the propeller revolution speed as a constant during the voyage first, then applying the modified isochrone method to determine the minimum time of passage. By varying propeller rotation speed this method is able to find the propeller rotation speed at which the minimum time of passage satisfies with the desired arrival time. This minimum time route will be treated as the minimum fuel route. Thus, the fuel consumption of this route itself is not minimized.

The 2DDP method based on Bellman's principle of optimality is similar to the modified isochrone method. It uses a recursive equation to solve ship 
routing problems formulated as a discrete optimization problem. The accuracy of the solution depends on the fineness of the grid system used. Compared with the modified isochrone method, the advantage of the 2DDP method is that it allows the operators to take into account of navigation boundaries by means of an appropriate selection of the grid points. Both the modified isochrone and 2DDP methods assume that ship sails at a constant propeller rotation speed or a constant engine power for the entire voyage.

The isopone method is an extension of the modified isochrone method. An isopone is the plane of equal fuel consumption that defines the outer boundary of the attainable region in a three-dimensional space, i.e. position and time. This method enables the operators to consider the variations of ship engine power to optimize the route. SPOS, a weather routing software, adopted the isopone method at the beginning of the software development. Although the isopone method is mathematically more elegant and theoretically offers better results than that of the modified isochrone method, finally SPOS had nevertheless abandoned the isopone method and applied the modified isochrone method. The main reason for this change was due to the fact that the isopone method appeared to be more difficult to understand by the operators onboard ships, whereas the modified isochrone method is straightforward and easy to understand.

Besides these methods, there are many other methods that have been used for weather routing in recently years, like iterative dynamic programming algorithm (Kyriakos Avgouleas 2008), augmented Lagrange multiplier (Masaru Tsujimoto, Katsuji Tanizawa 2006), Dijkstra algorithm (Chinmaya Prasad Padhy et al. 2008), genetic algorithm (Harries S, Hinnenthal J 2004) and so on.

Weather routing was first developed for determining shipping courses during a voyage with a minimum of passage time. However, nowadays shipping companies began to show more interesting in reducing fuel consumption driven by the fuel oil prices, environmental considerations and maintaining a certain time schedule which is specified in the chartering contract of a merchant vessel. In this paper, a new forward three dimensional dynamic programming (3DDP) method is presented for minimizing fuel consumption during a voyage. It is an extension of the traditional 2DDP method, allowing change heading and speed with both time and position, thus, it is able to realize a real global optimum result. Compared with the isopone method, the 3DDP method is straightforward and easy for programming.

\section{PROBLEM STATEMENT}

Ship engine power and shipping course directly decide the shipping route in the ocean. Ship speed over the ground depends on the engine power. There is a one-to-one relationship between them. Thus, both of them can be equally treated as the control variables in a weather routing process. In this paper, ship speed over the ground and shipping course measured from the true north are chosen as the control variables. The control variables are denoted as a control vector $\vec{U}, \vec{U}=U(u, \psi)$, where $u$ represents ship speed over the ground and $\psi$ is shipping course measured from the true north. Ship position $\vec{X}$ is also a vector, specified by longitude $\varphi$ and latitude $\theta$, $\vec{X}=X(\varphi, \theta)$.

Ship position $\vec{X}$ and voyage time $t$ determine the ship trajectory. Using $\vec{E}$ to denote weather conditions (speed and direction of wind, significant height, direction and peak frequency of wave and swell), $\vec{E}$ is a function of position $\vec{X}$ and time $t$,

$\vec{E}=E(\vec{X}, t)$

During a voyage, constraints $\vec{C}$ must be met. The constraints include geographic constraints, control constraints and safety constraints.

Thus, ship position $\vec{X}$ at time $t$ can be described by the function below:

$$
\vec{X}=f_{0}\left(\vec{X}^{\prime}, \vec{U}^{\prime}, \vec{E}^{\prime}, \vec{C}^{\prime}\right)
$$

Where $\vec{X}^{\prime}, \vec{U}^{\prime}, \vec{E}^{\prime}, \vec{C}^{\prime}$ correspond to time $t^{\prime}, t-$ $t^{\prime}=\Delta t, \Delta t$ is a time step used in calculation.

Because $\vec{E}^{\prime}$ is a function of $\vec{X}^{\prime}$ and $t^{\prime}$, so $\vec{X}$ can also be described by:

$$
\vec{X}=f\left(\vec{X}^{\prime}, \vec{U}^{\prime}, t^{\prime}, \vec{C}^{\prime}\right)
$$

This function can be explained that while comply with the constraints, the ship will arrive at the present position $\vec{X}$ at the present time $t$ from $\vec{X}^{\prime}$ under control of $\vec{U}^{\prime}$ during $\Delta t$ time step.

Instantaneous fuel consumption rate $q$ can be obtained by:

$$
q=q(\vec{X}, \vec{U}, t, \vec{C})
$$

The total fuel consumption for a voyage can be obtained by

$$
C=\int_{t_{\text {end }}}^{t_{s}} q(\vec{X}, \vec{U}, t, \vec{C}) d t
$$

Where:

Initial conditions: $\vec{X}_{s}=\left(\varphi_{s}, \theta_{s}\right), t=t_{s}$

Final conditions: $\vec{X}_{\text {end }}=\left(\varphi_{\text {end }}, \theta_{\text {end }}\right), t=t_{\text {end }}$ 
The constraints $\vec{C}$ : Geographic constraints, control constraints, safety constraints.

\section{DYNAMIC PROGRAMMING}

\subsection{Advantages of the 3DDP method}

Dynamic programming is a method which can solve complex problems by breaking them down into many simpler sub-problems. A stage is defined as the division of sequence of the sub-problems in the optimization procedure. The procedure of this method is to solve the sub-problems stage by stage. The variables used to define a stage must be parameters which are monotonically increasing with the progress of problem solving going-on. There are two choices of variable selection to specify a stage for ship routing problems, i.e. time and a measure of the progress of the vessel from departure (voyage progress). Each stage consists of many states which can be defined as a specific measurable condition of the ship operation, such as time and location. If time is chosen as the stage variable, the state can be defined by possible locations where the ship could pass. If voyage progress is chosen as the stage variable, states should be defined by time and possible positions away from the great circle.

The 2DDP method chose voyage progress as the stage variable. Because this method assumes that ships sail at a constant propeller rotation speed or a constant engine power for the entire voyage, there is a one-to-one relation between ship position and time. Thus, time variable is not needed to specify states in this method. Several authors have already attempted to solve the weather routing problem by using 3DDP treating both engine power and shipping course as the control variables during a voyage. Aligne, F. et al. (1998) chose time as the stage variable and used the forward algorithm; Henry Chen (1978) and Simon Calvert (1990) employed the voyage progress as the stage variable and used the backward algorithm. The method presented in this paper employs the voyage progress as the stage variable together with the use of the forward algorithm.

The advantages of using the forward algorithm can be stated as the following: When optimizing a route, the initial departure time is fixed, the arrival time can be treated as a flexible parameter, allowing a set of route with a minimum fuel consumption to be obtained corresponding to different specified arrival times in one calculation.

To compare using voyage time as stage variables, the advantages of using voyage progress as stage variables are:, ship headings are pre-defined by voyage progress on grid points, so that ship speed over the ground becomes the only explicitly defined control variable to be optimized during the routing op- timization process. This method doesn't need a finer grid system. It can save much more computing time than the methods which choose voyage time as a stage variable.

\subsection{Grid design}

Since the great circle is an optimum route under calm water conditions from the departure to the destination, it is chosen as a reference for the construction of the grid system used in the route optimization.

As describe above, states are of three dimensions, i.e. time and geographic location with a unit spacing $\Delta Y$ located on a stage, perpendicularly away from the great circles. The farthest states on a stage from the great circle are the possible locations the ship may pass to avoid a bad weather or certain sea

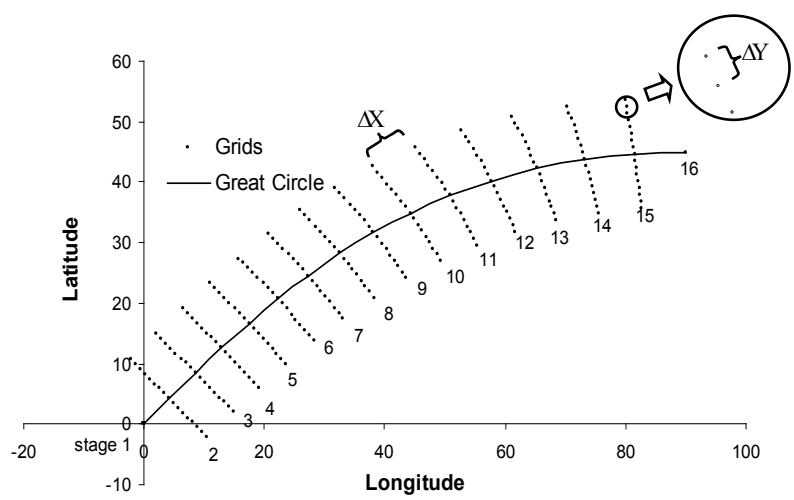

Figure 1. Projections of space grid system on a longitude $\times$ latitude plane.

conditions. Unlike the tradition dynamic programming, the variable of voyage time $t$ of states are determined as the optimization procedure is progressing. Grids should be deleted when a shipping route crosses islands/rocks.

Fig. 1 shows an example of stage projections on a longitude $\times$ latitude plane where 16 stages have been allocated $(1,2,3 \ldots 16)$ from the departure to the destination of a shipping route. The distance between two stages can be equally spaced $\Delta \mathrm{X}$. The total number of stages is determined according to the total distance of the route and the availability of computing capacity.

\subsection{Estimate of fuel consumption between two stages}

Fuel consumption is a function of ship hydrodynamics. The hydrodynamics of ship are modeled and simulated in the process of fuel consumption estimation. Thus, the accuracy of the modeling of the ship hydrodynamics is critical in the accuracy of fuel consumption estimation. As a consequence of added 
resistance due to wind and waves as well as the increased hull roughness over time, ship speed is often smaller than the designed speed so called involuntary speed reduction. Besides that, a voluntary speed reduction is needed to insure ship safety to minimize or avoid slamming, deck wetness, propeller racing, parametric rolling, motion sickness, engine overloading and so on. All these factors need to be considered in routing optimization as the constraints. Since the focus of this paper is to discuss the optimization algorithm of fuel consumption during a ship voyage, how to accurately predict ship hydrodynamic at the sea is not discussed in depth or further. However, the procedure of prediction of fuel consumption between two stages is presented here. This procedure can be treated as a sub-problem of a dynamic programming problem. The optimized fuel consumption during an entire voyage is obtained by adding up of all individual fuel consumption between two stages along a route with the newly developed 3DDP method.

As a ship voyage follows a predefined grid system, her heading is fixed between two stages. The ship speed over the ground is the only control variable which directly determines the fuel consumption between any two stages during a course of shipping.

Fig. 2 shows the procedure determining fuel consumption between two stages. In detail, it is as the following:

Step 1: Calculation of ship resistance. Ship resistance is calculated based on the ship speed over the ground, draft, trim and the weather condition. Ship resistance can be divided into three main components: a). the calm water resistance; b). the added resistance due to wave; c). the added resistance due to wind. Ship sea trial data, model test data and numerical simulation results are used to estimate these resistances.

Step 2: Estimation of engine power. The engine power is calculated to overcome the above calculated resistances based on the propeller characteristics.

Step 3: If the engine power is more than MCR (maximum continuous rate), the ship speed will be reduced by $\Delta u$, and then go back to step 1 .

Step 4: Calculation of probability of slamming, deck wetness, and propeller racing. To ensure the ship safety, if these constrain values exceed certain pre-set limits, the ship speed will be reduced $\Delta u$, and then go back to step 1 .

Step 5: Calculation of fuel consumption and ship position for next time interval $\Delta t$.

Step 6: Execute step 1 to step 5 repetitively in a fixed time interval $\Delta t$ between two stages until the ship (simulation step) arrives at the next stage or final destination.
The time interval $\Delta t$ for calculation is normally chosen at the frequency of the reception of weather forecasting onboard which is usually every 6 hours.

\subsection{Algorithm description}

The backward recursive algorithm has been used in most dynamic programming of weather routing. However, the forward dynamic programming offers more convenience in programming. The forward dynamic programming can be interpreted as that a path is optimal if and only if, for any intermediate stages, the choice of the foregoing path is optimum for this stage. By using this principle, the weather routing procedure can be broken down into a sequence of simpler problem solving. Notations defined in the programming are as follows.

- $K$ : total number of stage.

- $\quad N(k)$ : total number of state projection on the latitude $\times$ longitude plane on stage $k$, where: $k=1,2$, $3 \ldots K . N(1)=1, N(K)=1$.

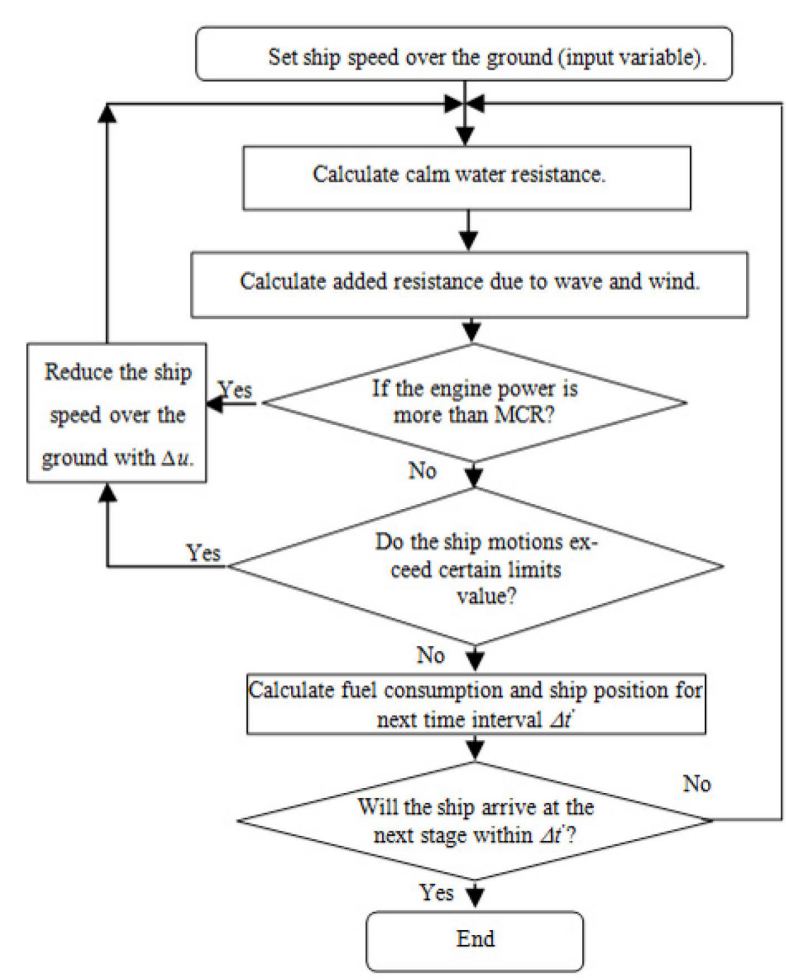

Figure 2. Estimate of fuel consumption between two stages.

- $P(i, k)$ : state project position on stage $k$, where: $i$ $=1,2,3 \ldots, N(k) . P(1,1)$ is the departure position; $P(1, K)$ is the destination position.

- $J$ : total number of time interval between states on a geographical position.

- $\Delta \bar{t}$ : time interval between states on a stage.

- $X(i, j, k)$ : a state on stage $k$, where: $i=1,2,3 \ldots$, $N(k), j=0,1,2 \ldots, J-1, k=1,2,3 \ldots, K$. The geographic position of the state $X(i, j, k)$ is $P(i$, $k$ ) on stage $k$, the time variable of the state is $t_{i, j, k}$, $\overline{t_{j}} \leqq t_{i, j, k} \leqq \bar{t}_{j+1}$, and $\overline{t_{j}}=\Delta \bar{t} \times j, \bar{t}_{j+1}=\Delta \bar{t} \times(j$ $+1)$. The state $X(i, j, k)$ at stage $k$ is a floating 
point between position $\left(P(i, k), \bar{t}_{j}\right)$ and $(P(i, k)$, $\left.\overline{t_{j+1}}\right)$.

- $X(1,0,1)$ : the initial state. Time variable $t_{1,0,1}$ of the initial state is 0 .

- $X(1, j, K)$ : the states on the final stage $K, j=0,1$, $2 \ldots, J-1$, the position of these states is $P(1, K)$.

- $F_{\text {opt }}(X(i, j, k))$ : the minimum fuel consumption from the initial state to the state $X(i, j, k)$.

- $u(m)$ : ship speed over the ground varying between 5 to 30 knot, $u(m)=5+0.1 \times(m-1)$, where: $m=1,2,3 \ldots, M$.

The recursion procedure of the forward dynamic programming can be described as follows:

Step 1: Set stage variable $k=1$.

Step 2: Iterate step 3 to 6 below for each attainable state $X^{*}=X(i, j, k)$ on stage $k$, where: $i=1,2$, $3 \ldots, N(k), j=0,1,2 \ldots, J$. if a state $X^{*}=X(i, j$, $k$ ) is unattainable due to constraints, its fuel consumption $F_{o p t}(X(i, j, k))$ is set to infinitive.

Step 3: Calculate ship heading $H^{*}$ from $X^{*}$ to the next stage position $P(i, k+1)$, where: $i^{\prime}=1,2$, $3 \ldots, N(k+1)$. Iterate step 4 to 5 for each $H^{*}$. If a ship heading $H^{*}$ violates the heading constraints or geographic feasibility, calculation for this heading is given up and go to the next heading calculation.

Step 4: Iterate steps 5 for each $u^{*}=u(m)$, where: $m=1,2,3 \ldots, M$. If certain ship speed $u^{*}$ violates the control constraints or safety constraints, skip out of this loop and go to the next loop.

Step 5: Choose $u^{*}$ and $H^{*}$ as the control variables, calculate the fuel consumption $\Delta f_{m . i}$, and the voyage time $\Delta t_{m, i}$, between state $X^{*}$ on stage $k$ and next state on stage $k+1$ with a geographic position $P(i, k+1)$ by using the method described in section $3.3, t_{m, i}$, is denoted as the arrival time at position $P(i, k+1)$ from the initial state, $t_{m, i},=t_{i, j, k}$ $+\Delta t_{m, i^{\prime}}$, the position $X^{\prime}=\left(P(i, k+1), t_{m, i}{ }^{\prime}\right)$ forms a new possible state on stage $k+1$, The fuel consumption at $X^{\prime}$ is $f^{*}$ is determined by $f^{*}=F_{\text {opt }}(X$ $(i, j, k))+\Delta f_{m, i}$.

Step 6: When the calculation of all possible states $X^{\prime}$ between time $\overline{t_{j^{\prime}}}$ and $\overline{t_{j^{\prime}+1}}$ at position $P(i, k+$ 1) on stage $k+1$ is completed, the possible state which has the minimum fuel consumption $f^{*}$ min is chosen as the state $X\left(i, j^{\prime}, k+1\right)$ Thus, $F_{\text {opt }}(X$ $\left.\left(i^{\prime}, j^{\prime}, k+1\right)\right)=f^{*}{ }_{\text {min }}$. The departure state $X^{*}$ on stage $k$, the arrival state $\left.X\left(i^{\prime}, j^{\prime}, k+1\right)\right)$ on stage $k+1$ and the corresponding control variables between the two states are saved for tracing the optimum route by a backward procedure at the end of the calculation. During the optimization process states within a time interval are floating. The benefit of using float states is that it eliminates the calculation of the interpolation. Thus, it can save computing time. When the weather in $\Delta \bar{t}$ time does not change much this method will not influence the accuracy of the optimized result.
Step 7: Let $k=k+1$, then go back to step 2 until $k$ $=K$.

Once the final state on stage $K$ has been obtained, a backward calculation procedure is used to identify the optimized fuel consumption route with the specified arrival time and the corresponding control variables during the entire voyage.

\section{CASE STUDY}

This section presents two case studies with the use of above described 3DDP method. As a simplification, the weather conditions are set artificially. Although the weather conditions used are not real and certain conditions may never happen in the reality, the use of artificial weather conditions will offer the same effect as the real ones in illustrating the methodology and advantages of the 3D dynamic programming. Holtrop method, a regression analysis method, is used to predict the total resistance in calm water. The engine power is calculated by propeller characteristics of the case ship.

Two different sets of weather conditions are used in the case studies with the following common parameters:

- Case ship: a 54,000 DWT container ship.

- Departure from: $\vec{X}_{s}=(0,0)$.

- Arrival at: $\vec{X}_{\text {end }}=(90,0)$.

- Time interval between states on a stage: $\Delta \bar{t}=1$ hour.

- Time step for fuel consumption calculation between two stages $\Delta t=6$ hours

- Ship speed: $u=5$ to 30 knots.

- Ship speed change step: $\Delta u=0.1$ knots.

- Total stage number: $K=16$.

- Total number of stage projection on a stage: $N(1)$ $=1, N(K)=1, N(k)=17$, where $k=2,3,4 \ldots K$ 1 .

- Stage space: $\Delta X=360$ nautical miles.

- State space: $\Delta Y=75$ nautical miles.

\subsection{Case study 1}

The geographic constraints and weather conditions for case 1 study are shown in Fig 3. The geographic constraints are set as a rectangular area which can be islands, rocks or mine fields. The scope of the geographic constraints is longitude from 50 to 70 degree and latitude from -1 to 7 degree. The envelop of the bad weather is set as a rectangular area as well, positioned longitude from 50 to 70 degree, latitude from - 1 to -9 degree at time $t=0$. The bad weather stays at this initial area for 60 hours before moving towards south with a speed of 3 knots. The ship is not allowed to enter into the bad weather area for safety consideration. Fig.4 shows the results of fuel con- 
sumption vs. time obtained from the route optimization for the case study 1 . When the specified arrival time is smaller than 233 hours, both of the 3DDP and 2DDP methods can get the similar strategies which choose the route closed to the dotted line in Fig. 5 and a constant ship speed during the voyage. That means, changing the ship heading can get much benefit than changing the ship speed at this weather condition. When the specified arrival time is more than 272 hours, the time during the voyage is relatively long, so the bad weather already pass away before the ship arrive there, the 3DDP method also get a similar strategy with the 2DDP method which choose the route closed to the solid line in Fig. 5 and a constant ship speed. When the specified arrival time is between 233 hours and 272 hours, the 3DDP method can get a better result than it calculated by the 2DDP method.

Fig. 5 and Fig. 6 show the optimized route and optimized ship speed obtained by using the 2DDP and 3DDP methods under a specified arrival time $t_{\text {end }}=264$ hours. The results have demonstrated that fuel consumption obtained by 2DDP is 1014.54 tons for the given voyage conditions and that is 969.25 tons if the ship follows the route resulted from 3DDP. As a result, route and operation profile optimized by the 3DDP offers about $4.5 \%$ of fuel saving compared that with the 2DDP. The reason for the fuel saving is that the 3DDP method permits the ship to change the heading and speed during the route. In the first section of the route, the ship slows down to let the bad weather pass-by first. Once the bad weather has passed, the ship increases her speed to ensure the desired arrival time is achieved.

\subsection{Case study 2}

In case 2 study the geographic constraints is the same as that of case 1 . Whereas the bad weather area at time $t=0$ is longitude from 50 to 70 degree, latitude from -7 to -15 degree. The bad weather moves towards north with a speed of 3 knots. Fig. 7 shows the geographic constraints and weather conditions. Fig. 8 shows results of fuel consumption vs. time obtained from the route optimization for the case study 2. Because of the same reasons with the case 1, when the specified arrival time is smaller than 270 hours or bigger than 303 hours, both the 3DDP and 2DDP methods can get the similar results; when the specified arrival time is between 270 hours and 303 hours, the 3DDP method can get a better result than it calculated by 2DDP method.

Fig. 9 and 10 present the route and ship speed optimized by the 2DDP and 3DDP methods under the same arrival time $t_{\text {end }}=278$ hours. The fuel consumption calculated by the 2DDP is 898 tons and that is 852 tons from the 3DDP calculation. A 5.1\% of fuel saving has been achieved by using the 3DDP compared with the 2DDP method. Unlike the case study 1 where ship speed is reduced to wait for the bad weather to pass during the first part of the route, the ship speed is increased to pass the region before the bad weather comes. Once the ship has passed the region where the bad weather is going to pass, the ship speed is slowed down and maintained the desired arrival time.

\section{CONCLUSION}

A newly developed 3DDP for weather routing has been presented. Case studies have shown that compared with the use of traditional 2DDP method, fuel saving can be achieved by using the newly developed 3DDP method in certain constraints and weather conditions. Since the speed of the ship varies according to the weather conditions and movement, the newly developed 3DDP increases the safety of shipping.

The 3DDP method considers optimization of both the ship speed and heading. Its operation and programming are easier and straight forward.

In this paper, real weather forecast is not considered, but this 3DDP method can also give enlightenment for the weather routing problem. In the future, this method will be used based on real weather forecast and ship hydrodynamics.

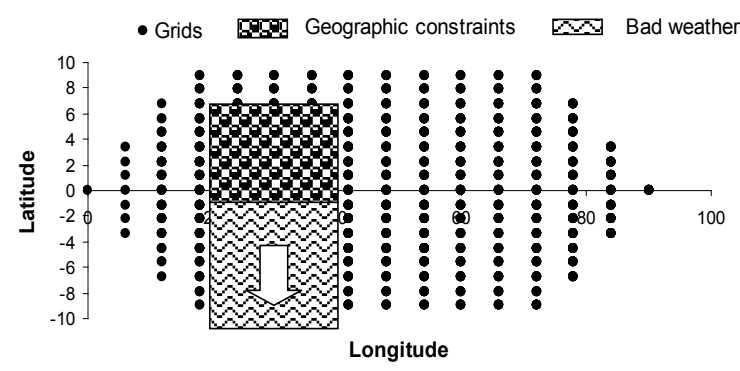

Figure 3. Geographic constraints and weather conditions (case $1)$.

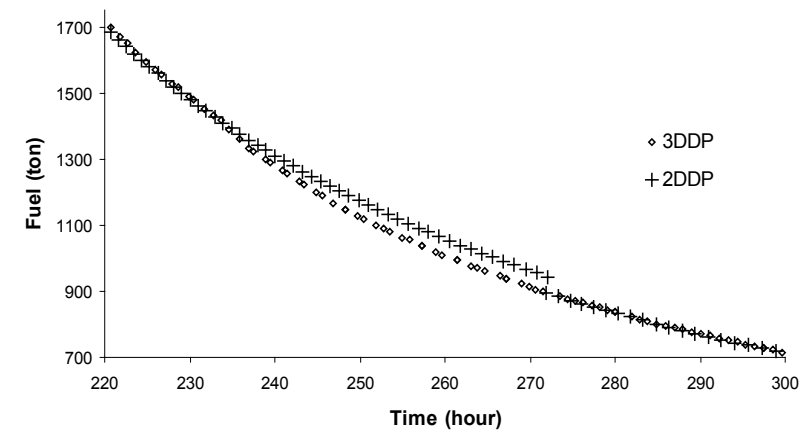

Figure 4. The fuel consumption vs. time curve (case 1). 


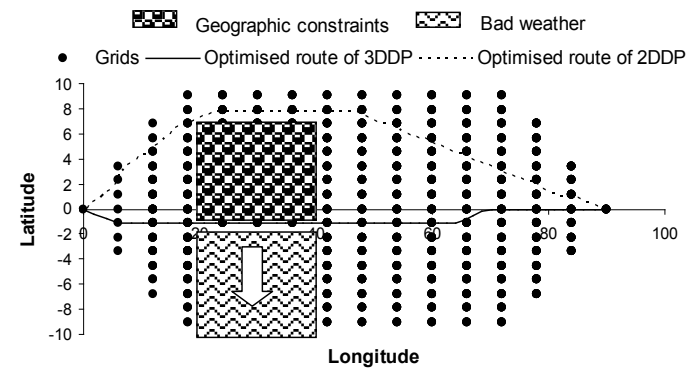

Figure 5. Optimized route (case 1).

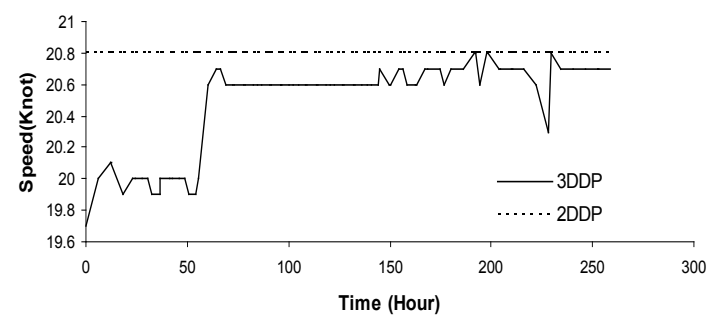

Figure 6. Optimized speed (case 1).

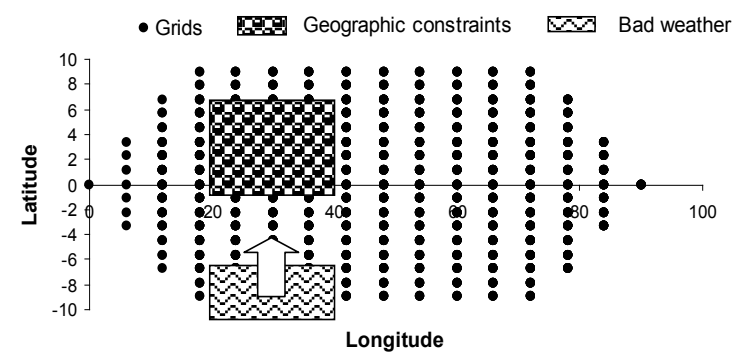

Figure 7. Geographic constraints and weather conditions (case 2).

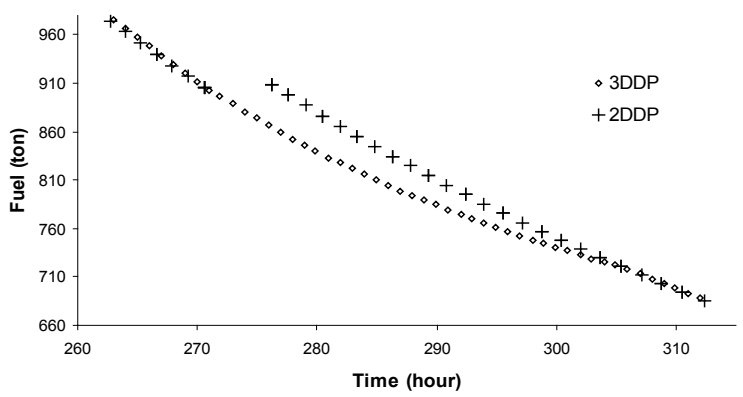

Figure 8. The fuel consumption vs. time curve (case 2).

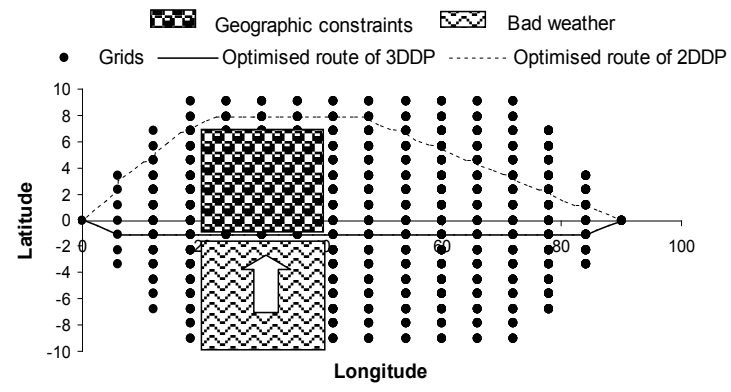

Figure 9. Optimized route (case 2).

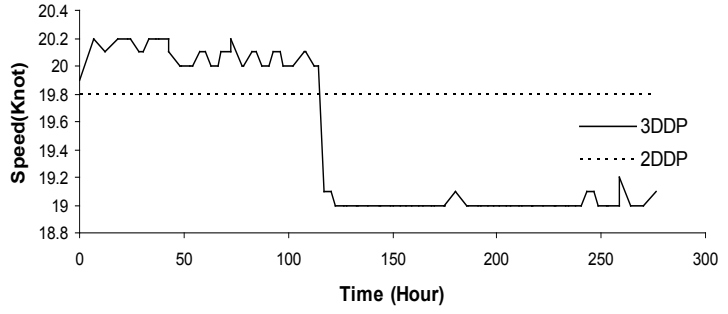

Figure 10. Optimized speed (case 2).

\section{REFERENCE}

Aligne F, Papageorgiou M, \& Walter E. 1998. Incorporating power variations into weather routing and why it may lead to better results. IFAC Control Applications in Marine Systems, Fukuoka, Japan, pp 269-274.

Bijlsma S.J. 1975. On minimal-time ship routing. $\mathrm{PhD}$ thesis. Royal Netherlands Meteorological Institute, Delft University of Technology.

Calvert S, Deakins E \& R. Motte. 1991. A dynamic system for fuel optimisation trans-ocean. Journal of Navigation 44(2): 233-265.

Calvert S. 1990. Optimal weather routeing procedures for vessels on trans-oceanic voyages. $\mathrm{PhD}$ thesis, Polytechnic South West.

Chen H. 1978. A dynamic program for minimum cost ship routing under uncertainty. PhD thesis, Dept. of Ocean Engineering, Massachusetts Institute of Technology.

Chinmaya Prasad Padhy, Debabrata Sen, \& Prasad Kumar Bhaskaran. 2008. Application of wave model for weather routing of ships in the North Indian Ocean. Natural Hazards 44(3): 373-385.

De Wit C. 1990. Proposal for low cost ocean weather routeing. Journal of Navigation 43(3): 428-439.

Hagiwara H \& Spaans JA. 1987. Practical weather routeing of sail-assisted motor vessels. Journal oF Navigation 40: 96119.

Hagiwara H. 1989. Weather routing of (sail-assisted) motor vessels. $\mathrm{PhD}$ thesis, Delft University of Technology.

Harries S, Hinnenthal J.2004. A systematic study on posing and solving the problem of pareto optimal ship routing. 3rd International Conference on Computer Applications and Information Technology in the Maritime Industries (COMPIT 2004), Siguenza, Spain.

Klompstra M.B, Olsde GJ \& Van Brunschot Pkgm. 1992. The isopone method in optimal control. Dynamics and Control 2(3):281-301.

Kyriakos Avgouleas. 2008. Optimal ship routing. Msc thesis, Massachusetts Institute of Technology.

Masaru Tsujimoto, Katsuji Tanizawa (2006) Development of a weather adaptive navigation system considering ship performance in actual seas. Proceedings of OMAE2006 $25^{\text {th }} \mathrm{In}$ ternational Conference on Offshore Mechanics and Arctic Engineering, Hamburg, Germany.

Spaans J.A. 1995. New developments in ship weather routing. Navigation 169: 95-106 